PROCEEDINGS OF THE

AMERICAN MATHEMATICAL SOCIETY

Volume 137, Number 3, March 2009, Pages 1001-1011

S 0002-9939(08)09679-2

Article electronically published on October 9, 2008

\title{
AN EXTENSION PROPERTY FOR THE FIGÀ-TALAMANCA HERZ ALGEBRA
}

\author{
CHRISTIAN FIORILLO
}

(Communicated by Nigel J. Kalton)

\begin{abstract}
Let $G$ be a locally compact group and $H$ a closed amenable subgroup of $G$. We prove that every element in $A_{p}(H)$ with compact support can be extended to an element of $A_{p}(G)$ of which we control the norm and support. The result is new even for the Fourier algebra. Our approach gives us new results concerning the operator norm closure of the convolution operators of $G$ with compact support.
\end{abstract}

\section{INTRODUCTION}

Let $G$ be a locally compact group and $H$ a closed subgroup of $G$. It is well-known that the restriction of $A_{p}(G)$ to $H$ is precisely $A_{p}(H)$ for every $1<p<\infty$. This deep result was obtained independently by C. Herz in [6] and by J.R. McMullen in [10. This extension property from $A_{p}(H)$ to $A_{p}(G)$ is important from the point of view of harmonic analysis. For instance the subgroup $H$ is a set of $p$-synthesis in $G$ if and only if there is a positive real number $C$ such that every $u \in A_{p}(H)$ with compact support has an extension $v$ in $A_{p}(G)$ with compact support and with

$$
\|v\|_{A_{p}(G)} \leq C\|u\|_{A_{p}(H)} .
$$

For $p \neq 2$ this problem is still open and far from being solved. Nevertheless, Herz (in the loco citato) solved this question for $p=2$ and for any $p$, assuming $H$ amenable or normal in $G$. In this last case, he obtained the $p$-synthesis of $H$ in a much stronger form: given an element of compact support $u \in A_{p}(H), \varepsilon>0$, and an open subset $U$ of $G$ with supp $u \subset U \cap H$, there is $v \in A_{p}(G)$ with $\|v\|_{A_{p}(G)}<\|u\|_{A_{p}(H)}+\varepsilon$, $\operatorname{Res}_{H} v=u$ and $\operatorname{supp} v \subset U$. We are able to obtain Herz's result for every closed amenable subgroup of $G$. However, we have to assume that both groups $G$ and $H$ are unimodular. Our result is new even for the Fourier algebras $A(H)$ and $A(G)$. We also construct a net of linear contractions $\left(\Gamma_{\alpha}\right)_{\alpha \in I}$ from $A_{p}(H)$ into $A_{p}(G)$ such that $\left(\operatorname{Res}_{H} \circ \Gamma_{\alpha}\right)_{\alpha \in I}$ converges to $i d_{A_{p}(H)}$, in the strong operator topology of $A_{p}(H)$. This last statement can be interpreted as a substitute of the nonexistence of liftings of $A_{p}(H)$ into $A_{p}(G)$ ([5]).

Received by the editors March 5, 2008

2000 Mathematics Subject Classification. Primary 43A07, 43A15, 43A22; Secondary 43A32, 43A45, 46J10.

Key words and phrases. Amenable groups, Figà-Talamanca Herz algebra, Fourier algebra, convolution operators.

(C)2008 American Mathematical Society Reverts to public domain 28 years from publication 
Let $c v_{p}(G)$ be the operator norm closure of all $p$-convolution operators with compact support. Recall that $c v_{2}(G)$ is isomorphic to $C_{u}^{b}(\widehat{G})$ for $G$ abelian. Our approach to the extension property allows us to show that $c v_{p}(H)$ is a retract of $c v_{p}(G)$ in the category BAN of Banach spaces (and their isometries). Herz obtained this result in 1970, assuming $G$ amenable and $H$ normal in $G$. Moreover, we also show that $c v_{p}(H)$ is isomorphic to $\left\{T \in c v_{p}(G) \mid \operatorname{supp} T \subset H\right\}$, a result that Herz also obtained assuming $H$ normal in $G$ ([7]).

The material presented in this paper is drawn from a section of the author's doctoral thesis 4 written at the EPFL (École Polytechnique Fédérale de Lausanne). The author wishes to express his deep gratitude to Professor Antoine Derighetti for kind advice and precious encouragement.

\section{DEFINITIONS AND PRELIMINARIES}

Let $G$ be a locally compact group, $H$ a closed subgoup of $G$ and $1<p<\infty$. Throughout this paper we will denote with $m_{G}$ (respectively $m_{H}$ ) the left Haar measure on $G$ (respectively on $H$ ). Moreover, we will indicate by $\mathscr{L}_{\mathbb{C}}^{p}(G)$ the Banach space $\left(\mathscr{L}_{\mathbb{C}}^{p}(G), m_{G}\right)$ of all $p$-integrable functions on $G$. For every $f \in \mathbb{C}^{G}$ and $x \in G$, we also denote $\overline{f(x)}$ by $\bar{f}(x)$ and $f\left(x^{-1}\right)$ by $\check{f}(x)$. We recall that the Banach algebra $A_{p}(G)$ can be described as follows:

$$
\begin{array}{r}
A_{p}(G)=\left\{u \in \mathbb{C}^{G} \mid \exists\left(\left(k_{n}\right)_{n=1}^{\infty},\left(l_{n}\right)_{n=1}^{\infty}\right) \in \mathcal{A}_{p}(G),\right. \\
\left.u(x)=\sum_{n=1}^{\infty} \bar{k}_{n} * \check{l}_{n}(x), \text { for every } x \in G\right\},
\end{array}
$$

where

$$
\begin{aligned}
\mathcal{A}_{p}(G) & =\left\{\left(\left(k_{n}\right)_{n=1}^{\infty},\left(l_{n}\right)_{n=1}^{\infty}\right) \mid \text { where }\left(k_{n}\right)_{n=1}^{\infty} \text { is a sequence of } \mathscr{L}_{\mathbb{C}}^{p}(G)\right. \text { and } \\
& \left.\left(l_{n}\right)_{n=1}^{\infty} \text { is a sequence of } \mathscr{L}_{\mathbb{C}}^{p^{\prime}}(G) \text { such that } \sum_{n=1}^{\infty} N_{p}\left(k_{n}\right) N_{p^{\prime}}\left(l_{n}\right)<\infty\right\},
\end{aligned}
$$

and for every $u \in A_{p}(H)$ :

$$
\begin{array}{r}
\|u\|_{A_{p}}(G):=\inf \left\{\sum_{n=1}^{\infty} N_{p}\left(k_{n}\right) N_{p^{\prime}}\left(l_{n}\right) \mid u=\sum_{n=1}^{\infty} \bar{k}_{n} * \check{l}_{n}\right. \\
\left.\quad \text { with }\left(\left(k_{n}\right)_{n=1}^{\infty},\left(l_{n}\right)_{n=1}^{\infty}\right) \in \mathcal{A}_{p}(G)\right\} .
\end{array}
$$

Let $C V_{p}(G)$ be the Banach algebra of $p$-convolution operators on $L^{p}(G)$. We denote by $P M_{p}(G)$ the ultraweak closure of all $p$-convolution operators $\lambda_{G}^{p}(\mu)^{1}$ defined by a bounded measure $\mu$ on $G$. We recall that, for $G$ amenable, $P M_{p}(G)=C V_{p}(G)$. Another well-known description of $P M_{p}(G)$ is as a dual of $A_{p}(G)$; the duality is

\footnotetext{
${ }^{1} \lambda_{G}^{p}(\mu)(\varphi)=\varphi *\left(\Delta_{G}^{-1 / p} \check{\mu}\right)$, where $\Delta_{G}$ is the modular function of $G$.
} 
given by

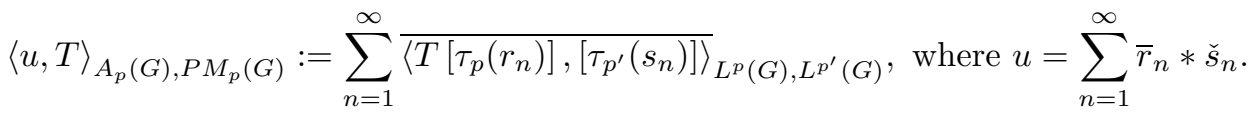

For every $\varphi \in \mathscr{L}_{\mathbb{C}}^{p}(G)$, we denote by $\tau_{p}(\varphi)=\Delta_{G}^{-1 / p} \check{\varphi}$ and by $[\varphi]$ the set all $\psi \in \mathbb{C}^{G}$ such that $\psi=\varphi$ a.e. We consider $\left(L_{\mathbb{C}}^{p}(G),\|\cdot\|_{p}\right)$ as the Banach space of all $[\varphi]$ with $\varphi \in \mathscr{L}_{\mathbb{C}}^{p}(G)$ and $\|\varphi\|_{p}=N_{p}(\psi)$ where $\psi \in[\varphi]$. Given $f \in C_{00}(G, \mathbb{C})$ and $\dot{x} \in G / H$, we define

$$
T_{H, q} f(\dot{x})=\int_{H} \frac{f(x h)}{q(x h)} d h,
$$

where $q(x h)=q(x) \Delta_{H}(h) \Delta_{G}\left(h^{-1}\right)$, for every $x \in G$ and $h \in H$ (we refer to [11]). For $k, l \in C_{00}(G, \mathbb{C})$ the relation

$$
\begin{aligned}
\left\langle\Lambda_{k, l}^{(q)}(T)[\varphi],[\psi]\right. & \rangle_{L_{\mathbb{C}}^{p}(H), L_{\mathbb{C}}^{p^{\prime}}(H)} \\
& :=\left\langle T\left[\tau_{p}\left(q^{1 / p}\left(k *_{H} \tau_{p} \varphi\right)\right)\right],\left[\tau_{p^{\prime}}\left(q^{1 / p^{\prime}}\left(l *_{H} \tau_{p^{\prime}} \psi\right)\right)\right]\right\rangle_{L_{\mathbb{C}}^{p}(G), L_{\mathbb{C}}^{p^{\prime}}(G)},
\end{aligned}
$$

with $\left(k *_{H} \tau_{p} \varphi\right)(x)=\int_{H} k(x h)\left(\tau_{p} \varphi\right)\left(h^{-1}\right) d h$, for every $x \in G$, defines a linear continuous map $\Lambda_{k, l}^{(q)}$ from $C V_{p}(G)$ into $C V_{p}(H)$. We have $\left\|\Lambda_{k, l}^{(q)}\right\| \leq\left\|T_{H}|k|\right\|_{p}\left\|T_{H}|l|\right\|_{p^{\prime}}$. Moreover, $\Lambda_{k, l}^{(q)}$ as a continuous map of $P M_{p}(G)$ into $P M_{p}(H)$ is the dual of a continuous map $\Phi_{k, l}^{(q)}$ of $A_{p}(H)$ into $A_{p}(G)$. We have

$$
\Phi_{k, l}^{(q)}(u)=\sum_{n=1}^{\infty} \overline{\left(q^{1 / p}\left(k *_{H} r_{n}\right)\right)} *_{G}\left(q^{1 / p^{\prime}}\left(l *_{H} s_{n}\right)\right)^{\vee} \quad \text { where } \quad u=\sum_{n=1}^{\infty} \bar{r}_{n} *_{s_{n}},
$$

and for every $x \in G$, we also have

$$
\Phi_{k, l}^{(q)}(u)(x)=\int_{H} \overline{\left(\tau_{p}\left(q^{1 / p} k\right)\right)} *\left(\tau_{p^{\prime}}\left({ }_{x^{-1}}\left(q^{1 / p^{\prime}} l\right)\right)\right)^{\vee}(h) \Delta_{H}^{-1 / p^{\prime}}(h) u(h) d h .
$$

Recall that $r_{x, H}(h)=r(x h)$ for every $r \in \mathbb{C}^{G}, x \in G$ and $h \in H$. We also recall that there is a canonical homomorphism $i$ of the Banach algebra $C V_{p}(H)$ into $C V_{p}(G)$. Moreover $i$ is the dual of $\operatorname{Res}_{H}$. In this paper, we always suppose that $q=1$, so we put $\Lambda_{k, l}^{(1)}=\Lambda_{k, l}, \Phi_{k, l}^{(1)}=\Phi_{k, l}$ and $T_{H, 1}=T_{H}$.

\section{An APPRoXimate theOREM OF CONVOLUtion OPERATORS OF A CLOSED SUBGROUP}

Lemma 1. Let $G$ be a locally compact unimodular noncompact group, $H$ a closed amenable subgroup, $K$ a compact subset of $H, \varepsilon>0$ and $U$ a neighborhood of $e$ in $G$. Then there exists a symmetric $m_{G}$-integrable subset $V$ of $G$ such that $V \subset U$, $m_{G}(V)>0$ and such that for every $x \in K$, we have

$$
\int_{G}\left|1_{V}(y)-1_{x V x^{-1}}(y)\right| d y<\varepsilon m_{G}(V) .
$$


Proof. We suppose $m_{H}(K)>0$. Let $K^{\prime}:=K \cup K^{2}$. According to Lemma 2.2 in [3], there exists a symmetric $m_{G}$-integrable subset $V$ of $G$ and an $m_{H}$-integrable subset $N$ of $H$ such that $V \subset U, m_{G}(V)>0, N \subset K^{\prime}$,

$$
2 m_{H}(N)<m_{H}(K)
$$

and, for every $x \in K^{\prime} \backslash N$, we have

$$
\int_{G}\left|1_{V}(y)-1_{x V x^{-1}}(y)\right| d y<\frac{\varepsilon}{2} m_{G}(V) .
$$

Let $M:=K^{\prime} \backslash N$. For every $x, y \in M$ we have

$$
\int_{G}\left|1_{V}(\xi)-1_{x y^{-1} V y x^{-1}}(\xi)\right| d \xi=\int_{G}\left|1_{x V x^{-1}}(\xi)-1_{y V y^{-1}}(\xi)\right| d \xi,
$$

so, by (2) we obtain that for every $x \in M M^{-1}$,

$$
\int_{G}\left|1_{V}(y)-1_{x V x^{-1}}(y)\right| d y<\varepsilon m_{G}(V) .
$$

It remains to prove that $K \subset M M^{-1}$. Given $k \in K$ it suffices to verify that $m_{H}((k M) \cap M)>0$. Since

$$
\left(k K^{\prime}\right) \cap K^{\prime} \subset\left(K^{\prime} \backslash M\right) \cup\left(k\left(K^{\prime} \backslash M\right)\right) \cup((k M) \cap M),
$$

by recalling that $N=K^{\prime} \backslash M$ and by (1), we get

$$
m_{H}\left(\left(k K^{\prime}\right) \cap K^{\prime}\right)<m_{H}(K)+m_{H}((k M) \cap M) .
$$

Clearly $m_{H}(K) \leq m_{H}\left(\left(k K^{\prime}\right) \cap K^{\prime}\right)$; hence the proof is completed.

Lemma 2. Let $G$ be a locally compact unimodular noncompact nondiscrete group, $H$ a closed amenable subgroup, $K$ a compact subset of $H, \varepsilon>0$ and $U$ a neighborhood of e in $G$.

Then there exists an open symmetric relatively compact neighborhood $V$ of $e$ in $G$ such that $V \subset U, m_{G}(V)>0$ and such that for every $x \in K$, we have

$$
\int_{G}\left|1_{V}(y)-1_{x V x^{-1}}(y)\right| d y<\varepsilon m_{G}(V) .
$$

Proof. Let $U_{0}$ be an open symmetric relatively compact neighborhood of $e$ in $G$ such that $U_{0} \subset U$. By the previous lemma, there is a symmetric $m_{G}$-integrable subset of $G$ such that $W \subset U_{0}, m_{G}(W)>0$ and, for every $x \in K$, we have

$$
\int_{G}\left|1_{W}(y)-1_{x W x^{-1}}(y)\right| d y<\frac{\varepsilon}{2} m_{G}(W) .
$$

Let $Z:=W \cup\{e\}$. There exists an open subset $U_{1}$ of $G$ such that $Z \subset U_{1}$ and

$$
\int_{G}\left|1_{U_{1}}(y)-1_{W}(y)\right| d y<\frac{\varepsilon}{4} m_{G}(W) .
$$

Let $V:=U_{1} \cap U_{1}^{-1} \cap U_{0}$. Clearly, $V$ is an open symmetric neighborhood of $e$ in $G$ with $V \subset U$. We have

$$
\int_{G}\left|1_{V}(y)-1_{Z}(y)\right| d y<\frac{\varepsilon}{4} m_{G}(W)
$$


and

$$
\begin{aligned}
& \frac{1}{m_{G}(V)} \int_{G}\left|1_{V}(y)-1_{x V x^{-1}}(y)\right| d y \\
& \quad \leq \frac{2}{m_{G}(Z)} \int_{G}\left|1_{V}(y)-1_{Z}(y)\right| d y+\frac{1}{m_{G}(W)} \int_{G}\left|1_{W}(y)-1_{x W x^{-1}}(y)\right| d y .
\end{aligned}
$$

The conclusion follows from (3) and (4).

Lemma 3. Let $G$ be a locally compact unimodular nondiscrete group, $H$ a closed amenable unimodular subgroup, $K$ a compact subset of $G, V$ an open neighborhood of $e$ in $G, 1<p<\infty$ and $\varepsilon>0$.

Then there exists $U$, an open symmetric neighborhood of e in $G$, relatively compact in $G$ such that $U \subset V$ and

$$
\int_{K}\left|\left(\left(T_{H} 1_{U}\right) \circ \omega\right)(x)-\left(\left(T_{H} 1_{U}\right) \circ \omega\right)^{\vee}(x)\right| d x \leq \varepsilon m_{G}(U),
$$

where $\omega$ is the canonical map of $G$ onto $G / H$.

Proof. Let $U_{0}$ be a compact symmetric neighborhood of $e$ in $G$ with $U_{0} \subset U$. By the previous lemma, there exists an open symmetric neighborhood of $e$ in $G$ such that $V \subset U_{0}$ and

$$
\int_{G}\left|1_{V h}(y)-1_{h V}(y)\right| d y<\frac{\varepsilon m_{G}(V)}{1+m_{H}\left(K_{H}\right)},
$$

for every $h \in K_{H}$, where $K_{H}:=\left(K U_{0} \cup U_{0} K\right) \cap H$.

For every $x \in K$ let

$$
A_{x}:=\left\{h \in H \mid 1_{V h}(x)-1_{h V}(x) \neq 0\right\} .
$$

Taking into account that $A_{x} \subset K_{H}$, we have

$$
\begin{aligned}
\int_{K}\left|\left(\left(T_{H} 1_{U}\right) \circ \omega\right)(x)-\left(\left(T_{H} 1_{U}\right) \circ \omega\right)^{\vee}(x)\right| d x \\
\leq \int_{H} 1_{K_{H}}(h)\left(\int_{G}\left|1_{V h}(y)-1_{h V}(y)\right| d y\right) d h .
\end{aligned}
$$

Then the conclusion follows from (5).

Lemma 4. Let $G$ be a locally compact group and $H$ a closed subgroup of $G$. We suppose that $\operatorname{Res}_{H}\left(\Delta_{G}\right)=\Delta_{H}$. Then, for $1<p<\infty, f \in C_{00}(H, \mathbb{C}), k \in$ $C_{00}(G, \mathbb{C})$ and $U$ an open relatively compact neighborhood of $e$ in $G$, the following inequality holds:

$$
N_{p}\left(\left(f *_{H} k\right)\left(\left(T_{H} 1_{U}\right) \circ \omega\right)^{1 / p}\right) \leq N_{p}(f) m_{G}(U)^{1 / p}\left\|T_{H}(|k|)\right\|_{\infty}^{1 / p}\left\|T_{H}(|\check{k}|)\right\|_{\infty}^{1 / p^{\prime}} .
$$

Proof. We have

$$
N_{1}\left(\left(\varphi *_{H} k\right)\left(\left(T_{H} 1_{U}\right) \circ \omega\right)\right) \leq m_{G}(U) N_{1}(\varphi)\left\|T_{H}(|k|)\right\|_{\infty}
$$

and

$$
\left\|\varphi *_{H} k\right\|_{\infty} \leq\|\varphi\|_{\infty}\left\|T_{H}(|\check{k}|)\right\|_{\infty} .
$$


It suffices to verify that for a step function $f$ on $H$ with $N_{p}(f)=1$ and for a step function $g$ on $G$ with $N_{p^{\prime}}(g)=1$ we have

$$
\left|\int_{G} g(x)\left(f *_{H} k\right)(x)\left(T_{H} 1_{U}(\omega(x))\right)^{1 / p} d x\right| \leq m_{G}(U)^{1 / p}\left\|T_{H}(|k|)\right\|_{\infty}^{1 / p}\left\|T_{H}\left(\left|\check{k}^{\prime}\right|\right)\right\|_{\infty}^{1 / p^{\prime}} .
$$

Let

$$
f=\sum_{j=1}^{m}\left|a_{j}\right| e^{i \theta_{j}} 1_{E_{j}} \text { and } g=\sum_{l=1}^{n}\left|b_{l}\right| e^{i \varphi_{l}} 1_{F_{l}}
$$

with $a_{1}, \ldots, a_{m}, b_{1}, \ldots, b_{n} \in(0, \infty), 0 \leq \theta_{j}<2 \pi, 0 \leq \varphi_{l}<2 \pi, E_{1}, \ldots, E_{m}$ disjoint integrable and $F_{1}, \ldots, F_{n}$ also disjoint integrable sets. For $z \in B$ where $B=$ $\{z \mid z \in \mathbb{C}, 0 \leq \Re(z) \leq 1\}$ we put

$$
\begin{gathered}
f_{(z)}=\sum_{j=1}^{m}\left|a_{j}\right|^{(1-z) p} e^{i \theta_{j}} 1_{E_{j}}, \\
g_{(z)}=\sum_{l=1}^{n}\left|b_{l}\right|^{z p^{\prime}} e^{i \varphi_{l}} 1_{F_{l}}, \\
T_{(z)} \varphi=\frac{\left(\left(T_{H} 1_{U}\right) \circ \omega\right)^{1-z}\left(\varphi *_{H} k\right)}{m_{G}(U)^{1-z}}
\end{gathered}
$$

for $\varphi \in \mathscr{L}_{\mathbb{C}}^{p}(H)$, and

$$
F(z)=\int_{G}\left(T_{(z)} f_{(z)}\right)(x) g_{(z)}(x) d x .
$$

Then $F$ is continuous on $B$ and analytic on the interior of $B$. Moreover $F$ is bounded on $B$; more precisely,

$$
\begin{aligned}
|F(z)| \leq & \frac{\left\|\left(T_{H} 1_{U}\right)\right\|_{\infty}}{\min \left\{1, m_{G}(U)\right\}} \\
& \times \sum_{j=1}^{m} \sum_{l=1}^{n} \max \left\{\left|a_{j}\right|^{p}, 1\right\} \max \left\{\left|b_{l}\right|^{p^{\prime}}, 1\right\}\left|\int_{G}\left(1_{E_{j}} *_{H} k\right)(x) 1_{F_{l}}(x) d x\right| .
\end{aligned}
$$

Letting $y \in \mathbb{R}$, we have

$$
|F(i y)| \leq\left\|g_{(i y)}\right\|_{\infty} N_{1}\left(T_{(i y)} f_{(i y)}\right)=\left\|g_{(i y)}\right\|_{\infty} \frac{N_{1}\left(\left(f_{(i y)} *_{H} k\right)\left(\left(T_{H} 1_{U}\right) \circ \omega\right)\right)}{m_{G}(U)} ;
$$

on the other hand,

$$
|F(1+i y)| \leq\left\|T_{(1+i y)} f_{(1+i y)}\right\|_{\infty} N_{1}\left(g_{(1+i y)}\right)=\left\|f_{(1+i y)}\right\|_{\infty} N_{1}\left(g_{(1+i y)}\right) .
$$

Using (6), (77) and observing that

$$
N_{1}\left(f_{(i y)}\right)=N_{p}(f)^{p}=1=N_{1}\left(g_{(1+i y)}\right)=N_{p^{\prime}}(g)^{p^{\prime}}
$$

and that

$$
\left\|g_{(i y)}\right\|_{\infty}=1=\left\|f_{(1+i y)}\right\|_{\infty}
$$

by the Three Lines Theorem we obtain $|F(1-1 / p)| \leq\left\|T_{H}(|k|)\right\|_{\infty}^{1 / p}\left\|T_{H}(|\check{k}|)\right\|_{\infty}^{1 / p^{\prime}}$. Then (8) holds. 
Theorem 1. Let $G$ be a locally compact unimodular group, $H$ a closed amenable unimodular subgroup of $G$ and $m \in \mathbb{N}$. Let $1<p<\infty, \varepsilon>0, U$ a neighborhood of e in $G$ and $\left(\left(\varphi_{n}{ }^{(j)}\right)_{n=1}^{\infty},\left(\psi_{n}{ }^{(j)}\right)_{n=1}^{\infty}\right) \in \mathcal{A}_{p}(H)$, for $1 \leq j \leq m$.

Then there exist $k, l \in C_{00}^{+}(G)$ with the following properties:

(i) $\operatorname{supp} k, \operatorname{supp} l \subset U$ and $\left\|\Lambda_{k, l}\right\| \leq 1$,

(ii) for every $S \in C V_{p}(H)$ and $j=1, \ldots, m$,

$$
\begin{aligned}
\sum_{n=1}^{\infty} \mid\left\langle\Lambda_{k, l}(i(S))\left[\varphi_{n}^{(j)}\right],\left[\psi_{n}^{(j)}\right]\right\rangle_{L^{p}(H), L^{p^{\prime}}(H)} & \\
& -\left\langle S\left[\varphi_{n}^{(j)}\right],\left[\psi_{n}^{(j)}\right]\right\rangle_{L^{p}(H), L^{p^{\prime}}(H)} \mid \leq \varepsilon\|S\| \|_{p} .
\end{aligned}
$$

Proof. If $G$ is compact or discrete, then $H$ is neutral in $G$, so the theorem is true ([2], Proposition 2, p. 1429). So we suppose $G$ is noncompact and nondiscrete. We also suppose $\varepsilon \leq 1$.

There exists for every $1 \leq j \leq m$ and every $n \in \mathbb{N}, r_{n}^{(j)}, s_{n}^{(j)} \in C_{00}(H, \mathbb{C})$ with

$$
N_{p}\left(\varphi_{n}^{(j)}-r_{n}^{(j)}\right)<\frac{\varepsilon}{3 \cdot 2^{n+2}\left(1+N_{p^{\prime}}\left(\psi_{n}^{(j)}\right)\right)}
$$

and

$$
N_{p^{\prime}}\left(\psi_{n}^{(j)}-s_{n}^{(j)}\right)<\frac{\varepsilon}{3 \cdot 2^{n+2}\left(1+N_{p}\left(\varphi_{n}^{(j)}\right)\right)} .
$$

We can also find $N \in \mathbb{N}$ with

$$
\sum_{n=N+1}^{\infty} N_{p}\left(r_{n}^{(j)}\right) N_{p^{\prime}}\left(s_{n}^{(j)}\right)<\frac{\varepsilon}{2^{5}}
$$

for every $j=1, \ldots, m$.

Let $U_{0}$ be a symmetric compact neighborhood of $e$ in $G$ such that $U_{0} \subset U$. There exists $V$, a neighborhood of $e$ in $H$, such that

$$
N_{p}\left(r_{n}^{(j)}-\left(r_{n}^{(j)}\right)_{h^{-1}}\right)<\frac{\varepsilon_{1}}{2} \quad \text { and } \quad N_{p^{\prime}}\left(s_{n}^{(j)}-\left(s_{n}^{(j)}\right)_{h^{-1}}\right)<\frac{\varepsilon_{1}}{2}
$$

for every $h \in V, 1 \leq n \leq N, 1 \leq j \leq m$, where

$$
\varepsilon_{1}<\min \left\{\frac{\varepsilon}{3 \cdot 2^{n+2}\left(1+N_{p}\left(r_{n}^{(j)}\right)+N_{p^{\prime}}\left(s_{n}^{(j)}\right)\right)} \mid 1 \leq n \leq N, 1 \leq j \leq m\right\} .
$$

Choose $k^{\prime} \in C_{00}^{+}(G)$ such that $\operatorname{supp} k^{\prime} \subset U_{0},\left(\operatorname{supp} k^{\prime} \cap H\right) \subset V$,

$$
\int_{H} k^{\prime}(h) d h=1, \int_{H} k^{\prime}(x h) d h \leq 1 \text { and } \int_{H} k^{\prime}(h x) d h \leq 1 \text { for every } x \in G .
$$

So we obtain

$$
N_{p}\left(r_{n}^{(j)}-\operatorname{Res}_{H}\left(r_{n}^{(j)} *_{H} k^{\prime}\right)\right)<\frac{\varepsilon_{1}}{2} \text { and } N_{p^{\prime}}\left(s_{n}^{(j)}-\operatorname{Res}_{H}\left(s_{n}^{(j)} *_{H} k^{\prime}\right)\right)<\frac{\varepsilon_{1}}{2} .
$$

Let $U_{1}$ be a relatively compact neighborhood of $e$ in $G$ such that for every $x \in U_{1}$, for every $1 \leq n \leq N$, and for every $1 \leq j \leq m$,

$$
N_{p}\left(\left(r_{n}^{(j)} *_{H} k^{\prime}\right)_{x, H}-\operatorname{Res}_{H}\left(r_{n}^{(j)} *_{H} k^{\prime}\right)\right)<\frac{\varepsilon_{1}}{2}
$$


and

$$
N_{p^{\prime}}\left(\left(s_{n}^{(j)} *_{H} k^{\prime}\right)_{x, H}-\operatorname{Res}_{H}\left(s_{n}^{(j)} *_{H} k^{\prime}\right)\right)<\frac{\varepsilon_{1}}{2} .
$$

By Lemma 3 there exists $U_{2}$, an open symmetric neighborhood of $e$ in $G$ such that $U_{2} \subset U_{0} \cap U_{1}$ and

$$
\int_{K U_{0}}\left|T_{H} 1_{U_{2}}(\omega(x))-T_{H} 1_{U_{2}}\left(\omega\left(x^{-1}\right)\right)\right| d x<\varepsilon_{2} m_{G}\left(U_{2}\right),
$$

where

$$
K=\bigcup_{j=1}^{m}\left\{\left(\bigcup_{n=1}^{N} \operatorname{supp}\left(r_{n}^{(j)}\right)\right) \cup\left(\bigcup_{n=1}^{N} \operatorname{supp}\left(s_{n}^{(j)}\right)\right)\right\}
$$

and

$$
\varepsilon_{2}<\min \left\{\frac{\varepsilon_{1}^{p}}{A_{n j}^{p}}, \frac{\varepsilon_{1}^{p^{\prime}}}{B_{n j}^{p^{\prime}}} \mid 1 \leq n \leq N, 1 \leq j \leq m\right\}
$$

with

$$
A_{n j}=2^{n+3}\left(1+\left\|r_{n}^{(j)} *_{H} k^{\prime}\right\|_{\infty}\right)\left(1+N_{p^{\prime}}\left(s_{n}^{(j)}\right)\right)
$$

and

$$
B_{n j}=2^{n+3}\left(1+\left\|s_{n}^{(j)} *_{H} k^{\prime}\right\|_{\infty}\right)\left(1+N_{p^{\prime}}\left(r_{n}^{(j)}\right)\right) .
$$

We set

$$
k^{\prime \prime}=\frac{\left((\check{r})^{1 / p} k^{\prime}\right)^{\vee}}{m_{G}\left(U_{2}\right)^{1 / p}} \quad \text { and } \quad l^{\prime \prime}=\frac{\left((\check{r})^{1 / p^{\prime}} k^{\prime}\right)^{\vee}}{m_{G}\left(U_{2}\right)^{1 / p^{\prime}}} \quad \text { with } \quad r=\left(T_{H} 1_{U_{2}}\right) \circ \omega .
$$

Then for every $1 \leq n \leq N, 1 \leq j \leq m$,

$$
\begin{aligned}
\mid\left\langle\Lambda_{k^{\prime \prime}, l^{\prime \prime}}(i(S))\left[r_{n}^{(j)}\right],\left[s_{n}^{(j)}\right]\right\rangle_{L_{\mathbb{C}}^{p}(H), L_{\mathbb{C}}^{p^{\prime}}(H)} & \\
- & \left\langle S\left[r_{n}^{(j)}\right],\left[s_{n}^{(j)}\right]\right\rangle_{L_{\mathbb{C}}^{p}(H), L_{\mathbb{C}}^{p^{\prime}}(H)} \mid \leq \frac{5 \varepsilon\|S\| \|_{p}}{2^{5}} .
\end{aligned}
$$

Now choose $f, g \in C_{00}^{+}(G / H)$ with

$$
N_{p}\left(f-\left(\frac{\dot{r}^{1 / p}}{m_{G}\left(U_{2}\right)^{1 / p}}\right)\right)<\varepsilon_{3} \quad \text { and } \quad N_{p^{\prime}}\left(g-\left(\frac{\dot{r}^{1 / p^{\prime}}}{m_{G}\left(U_{2}\right)^{1 / p^{\prime}}}\right)\right)<\varepsilon_{3}
$$

with

$$
\varepsilon_{3}<\min \left\{\frac{\varepsilon}{C_{j}} \mid 1 \leq j \leq m\right\} \text { where } C_{j}=2^{6}\left(1+\sum_{n=1}^{\infty} N_{p}\left(r_{n}^{(j)}\right) N_{p^{\prime}}\left(s_{n}^{(j)}\right)\right) .
$$

To finish the proof it suffices to take

$$
k=\frac{(f \circ \omega)\left(k^{\prime}\right)^{\vee}}{1+\varepsilon_{3}} \quad \text { and } \quad l=\frac{(g \circ \omega)\left(k^{\prime}\right)^{\vee}}{1+\varepsilon_{3}} .
$$




\section{Extension theorem for the Figà-Talamanca Herz algebra}

Corollary 1. Let $G$ be a locally compact unimodular group, $H$ a closed amenable unimodular subgroup of $G$ and $1<p<\infty$. Let $u \in A_{p}(H) \cap C_{00}(H ; \mathbb{C}), \varepsilon>0$ and $U$ an open subset of $G$. Suppose that $\operatorname{supp} u \subset U$. Then, there exists $v \in A_{p}(G) \cap$ $C_{00}(G ; \mathbb{C})$ with $\operatorname{supp} v \subset U,\|v\|_{A_{p}(G)} \leq\|u\|_{A_{p}(H)}$ and $\left\|u-\operatorname{Res}_{H} v\right\|_{A_{p}(H)} \leq \varepsilon$.

Proof. There are sequences $\left(r_{n}\right)_{n=1}^{\infty},\left(s_{n}\right)_{n=1}^{\infty}$ of $C_{00}(H ; \mathbb{C})$ with $\sum_{n=1}^{\infty} N_{p}\left(r_{n}\right) N_{p^{\prime}}\left(s_{n}\right)$ $<\infty$ and $u=\sum_{n=1}^{\infty} \bar{r}_{n} * \check{s}_{n}$. By Theorem 1 , there exist $k, l \in C_{00}^{+}(G)$ with $\operatorname{supp} k$, $\operatorname{supp} l \subset U_{0},\left\|\Lambda_{k, l}\right\| \leq 1$ and with

$$
\sum_{n=1}^{\infty}\left|\left\langle\Lambda_{k, l}(i(S))\left[\check{r}_{n}\right],\left[\check{s}_{n}\right]\right\rangle_{L_{\mathbb{C}}^{p}(H), L_{\mathbb{C}}^{p^{\prime}}(H)}-\left\langle S\left[\check{r}_{n}\right],\left[\check{s}_{n}\right]\right\rangle_{L_{\mathbb{C}}^{p}(H), L_{\mathbb{C}}^{p^{\prime}}(H)}\right| \leq \varepsilon\left\|\left|\|S \mid\|_{p}\right.\right.
$$

for every $S \in C V_{p}(H)$, where $U_{0}$ is an open neighborhood of $e$ in $G$ such that $U_{0} \operatorname{supp} u U_{0}^{-1} \subset U$.

The function $\Phi_{k, l}(u)$ satisfies the required properties.

The main result of this work is the following.

Theorem 2. Let $G$ be a locally compact unimodular group, $H$ a closed amenable unimodular subgroup of $G$ and $1<p<\infty$. Let $u \in A_{p}(H) \cap C_{00}(H ; \mathbb{C}), \varepsilon>0$ and $U$ an open subset of $G$. Suppose that $\operatorname{supp} u \subset U$. Then, there exists $v \in$ $A_{p}(G) \cap C_{00}(G ; \mathbb{C})$ with $\operatorname{supp} v \subset U,\|v\|_{A_{p}(G)}<\|u\|_{A_{p}(H)}+\varepsilon$ and $\operatorname{Res}_{H} v=u$.

Proof. Let $U^{\prime}$ be an open relatively compact subset of $G$ with supp $u \subset U^{\prime} \subset \overline{U^{\prime}} \subset$ $U$. We use Corollary 1 to define by induction a sequence $\left(h_{n}\right)_{n=1}^{\infty}$ of $A_{p}(H) \cap$ $C_{00}(H ; \mathbb{C})$ and a sequence $\left(g_{n}\right)_{n=1}^{\infty}$ of $A_{p}(G) \cap C_{00}(G ; \mathbb{C})$ with:

$$
\begin{aligned}
& \text { (1) } h_{1}=u, \\
& \text { (2) } \operatorname{supp}\left(h_{n}\right) \subset U^{\prime} \cap H \text { and } \operatorname{supp}\left(g_{n}\right) \subset U^{\prime} \\
& \text { (3) }\left\|g_{n}\right\|_{A_{p}(G)} \leq\left\|h_{n}\right\|_{A_{p}(H)}, \\
& \text { (4) }\left\|\operatorname{Res}_{H}\left(g_{n}\right)-h_{n}\right\|_{A_{p}(H)}<\frac{\varepsilon}{2^{n+1}} \\
& \text { (5) } h_{n+1}=h_{n}-\operatorname{Res}_{H}\left(g_{n}\right) .
\end{aligned}
$$

The function $\sum_{n=1}^{\infty} g_{n}$ has the desired properties.

\section{Applications}

Theorem 3. Let $G$ be a locally compact unimodular group, $H$ a closed amenable unimodular subgroup of $G$ and $1<p<\infty$. There exists a linear contraction $\mathcal{P}$ from $\mathscr{L}\left(L_{\mathbb{C}}^{p}(G)\right)$ to $\mathscr{L}\left(L_{\mathbb{C}}^{p}(H)\right)$ with the following properties:

(i) $\mathcal{P}(T) \in C V_{p}(H)$, for every $T \in C V_{p}(G)$;

(ii) $\mathcal{P}(i(S))=S$, for every $S \in C V_{p}(H)$;

(iii) $\operatorname{supp} \mathcal{P}(T) \subset \operatorname{supp} T$, for every $T \in C V_{p}(G)$. 
Proof. Consider the set $\mathscr{F}$ of all maps $F$ of $\mathscr{L}\left(L_{\mathbb{C}}^{p}(G)\right) \times L_{\mathbb{C}}^{p}(H) \times L_{\mathbb{C}}^{p^{\prime}}(H)$ into $\mathbb{C}$, linear in the first two variables and antilinear in the third with $|F(T, \varphi, \psi)| \leq$ $C \mid\|T\|_{p}\|\varphi\|_{p}\|\psi\|_{p^{\prime}}$ for some $C \in[0, \infty)$, depending on $F$. We put

$$
\|F\|=\sup \left\{|F(T, \varphi, \psi)|\left|\|T \mid\|_{p} \leq 1,\|\varphi\|_{p} \leq 1,\|\psi\|_{p^{\prime}} \leq 1\right\} \text {, for every } F \in \mathscr{F},\right.
$$

and for $k, l \in C_{00}(G ; \mathbb{C})$ we set

$$
F_{k, l}(T, \varphi, \psi)=\left\langle\Lambda_{k, l}(T) \varphi, \psi\right\rangle_{L_{\mathbb{C}}^{p}(H), L_{\mathbb{C}}^{p^{\prime}}(H)} .
$$

We have $F_{k, l} \in \mathscr{F}$.

Let $A$ be a finite subset of $\mathcal{A}_{p}(H), B$ a finite subset of $C V_{p}(H), U$ an open neighborhood of $e$ in $G$ and $\varepsilon>0$. Then Theorem 1 implies that the following set is nonempty:

$$
\begin{aligned}
K_{A, B, U, \varepsilon}:= & \left\{F_{k, l} \mid k, l \in C_{00}^{+}(G),\left\|F_{k, l}\right\| \leq 1, \operatorname{supp} k, \operatorname{supp} l \subset U,\right. \\
& \sum_{n=1}^{\infty}\left|F_{k, l}\left(i(S),\left[r_{n}\right],\left[s_{n}\right]\right)-\left\langle S\left[r_{n}\right],\left[s_{n}\right]\right\rangle_{L_{\mathbb{C}}^{p}(H), L_{\mathbb{C}}^{p^{\prime}}(H)}\right|<\varepsilon,
\end{aligned}
$$

for every $\left(\left(r_{n}\right)_{n=1}^{\infty},\left(s_{n}\right)_{n=1}^{\infty}\right) \in A$, for every $\left.S \in B\right\}$.

Let $\bar{K}_{A, B, U, \varepsilon}$ be the closure of $K_{A, B, U, \varepsilon}$ in $\mathscr{F}$ with respect to the topology $\sigma\left(\mathscr{F}, \mathscr{L}\left(L_{\mathbb{C}}^{p}(G)\right) \times L_{\mathbb{C}}^{p}(H) \times L_{\mathbb{C}}^{p^{\prime}}(H)\right)$. Choose

$$
J \in \bigcap\left\{\bar{K}_{A, B, U, \varepsilon} \mid A \text { a finite subset of } \mathcal{A}_{p}(H),\right.
$$

$$
B \text { a finite subset of } C V_{p}(H) \text {, }
$$

$$
U \text { an open neighborhood of } e \text { in } G \text { and } \varepsilon>0\} \text {. }
$$

The unique map $\mathcal{P}$ from $\mathscr{L}\left(L_{\mathbb{C}}^{p}(G)\right)$ to $\mathscr{L}\left(L_{\mathbb{C}}^{p}(H)\right)$ with $\langle\mathcal{P}(T) \varphi, \psi\rangle_{L_{\mathbb{C}}^{p}(H), L_{\mathbb{C}}^{p^{\prime}}(H)}=$ $J(T, \varphi, \psi)$ is linear and satisfies (i), (ii) and (iii).

Corollary 2. Let $G$ be a locally compact unimodular group, $H$ a closed amenable unimodular subgroup of $G$ and $1<p<\infty$. Then, via $i, c v_{p}(H)$ is isomorphic to $\left\{T \in c v_{p}(G) \mid \operatorname{supp} T \subset H\right\}$.

Remark 1. For $p=2$ we obtain a proof of a result announced by E. Kaniuth and A. Lau ([8, Lemma 3.2 (ii)).

Corollary 3. Let $G$ be a locally compact unimodular group, $H$ a closed amenable unimodular subgroup of $G$ and $1<p<\infty$. Then, $c v_{p}(H)$ is a retract in BAN of $c v_{p}(G)$.

Corollary 4. Let $G$ be a locally compact unimodular group, $H$ a closed amenable unimodular subgroup and $1<p<\infty$. Then there exists a net of morphisms $\left(\Gamma_{\alpha}\right)_{\alpha \in I}$ in $B A N$ from $A_{p}(H)$ into $A_{p}(G)$ with

$$
\lim _{\alpha \in I}\left\|\operatorname{Res}_{H}\left(\Gamma_{\alpha}(u)\right)-u\right\|_{A_{p}(H)}=0,
$$

for every $u \in A_{p}(H)$. 
Proof. On $\mathcal{I}=\left\{(E, \varepsilon) \mid E\right.$ a finite subset of $\left.A_{p}(H), \varepsilon>0\right\}$, the relation $(E, \varepsilon) \leq$ $\left(E^{\prime}, \varepsilon^{\prime}\right)$ defined by $E^{\prime} \subset E$ and $\varepsilon^{\prime}<\varepsilon$ is a filtering partial order. By Theorem 1 the set

$$
D(E, \varepsilon)=\left\{\Phi_{k, l} \mid k, l \in C_{00}^{+}(G),\left\|\Phi_{k, l}\right\| \leq 1,\left\|\operatorname{Res}_{H}\left(\Phi_{k, l}(u)\right)-u\right\|_{A_{p}(H)}<\varepsilon,\right.
$$

$$
\text { for every } u \in E\}
$$

is nonempty. Let $\Gamma$ be a map of $\mathcal{I}$ into $\bigcup_{(E, \varepsilon) \in \mathcal{I}} D(E, \varepsilon)$ with $\Gamma(E, \varepsilon) \in D(E, \varepsilon)$ for every $(E, \varepsilon) \in \mathcal{I}$.

The net $\Gamma$ satisfies the required properties.

Remark 2. If $H$ is metrizable and $\sigma$-compact, it is possible to replace this net by a sequence.

Remark 3. If $G$ is a locally compact group and $H$ a closed subgroup of $G$, then the restriction map $R: B(G)$ to $B(H)$ need not be surjective when $G$ is amenable, where $B(G)$ is the Fourier-Stieltjes algebra of $G$ which contains $A_{2}(G)=A(G)$, the Fourier algebra of $G$ being a closed ideal. For $G$ connected, surjectivity of $R$ is equivalent to $G$ being a SIN-group (see [1] and [9]).

\section{REFERENCES}

[1] M. Cowling and P. Rodway, Restrictions of certain function spaces to closed subgroups of locally compact groups, Pacific J. Math. 80 (1979), 91-104. MR.534697(80i:43008)

[2] J. Delaporte and A. Derighetti, Invariant projections and convolution operators, Proc. Amer. Math. Soc. 129 (2001), 1427-1435. MR1814169 (2002m:43002)

[3] A. Derighetti, On Herz's projection theorem, Illinois J. Math. 48 (2004), 463-476. MR2085420 (2006h:43002)

[4] C. Fiorillo, Extrapolation et synthèse, Ph.D. thesis, EPFL, Lausanne (2007).

[5] B. Forrest, Complemented ideals in the Fourier algebra and the Radon Nikodým property, Trans. Amer. Math. Soc. 333 (1992), 689-700. MR.1112546 (92m:43003)

[6] C. Herz, Harmonic synthesis for subgroups, Annales de l'Institut Fourier, tome 23, no. 3, Grenoble (1973), 91-123. MR0355482 (50:7956)

[7] Synthèse spectrale pour les sous-groupes par rapport aux algèbres $A_{p}$, C. R. Acad. Sci. Paris Sér. A-B 271, 1970, A316-A318. MR0273429 (42:8307b)

[8] E. Kaniuth and A. Lau, Spectral synthesis for $A(G)$ and subspaces of $V N(G)$, Proc. Amer. Math. Soc. 129 (2001), 3253-3263. MR1845000(2002h:43003)

[9] Extension and separation properties of positive definite functions on locally compact groups, Trans. Amer. Math. Soc. 359 (2007), 447-463. MR2247899 (2007k:43008)

[10] J.R. McMullen, Extensions of positive-definite functions, Memoirs of the American Mathematical Society 117 (1972), 1-71. MR0445229 (56:3573)

[11] H. Reiter and J.D. Stegeman, Classical harmonic analysis and locally compact groups, The Clarendon Press, Oxford University Press, New York (2000). MR.1802924 (2002d:43005)

Department of Mathematics, École Polytechnique Federale de Lausanne, Station 8, CH-1015 Lausanne, Switzerland

Current address: Via Pratocarasso 31, 6500 Bellinzona (TI), Switzerland 